\title{
Dexamethasone Downregulates Expressions of 14-3-3 $\beta$ and $\gamma$-Isoforms in Mice with Eosinophilic Meningitis Caused by Angiostrongylus cantonensis Infection
}

\author{
Hung-Chin Tsai ${ }^{1,2, *}$, Yu-Hsin Chen ${ }^{1}$, Chuan-Min Yen², Li-Yu Chung², Shue-Ren Wann ${ }^{1}$, Susan Shin-Jung Lee', \\ Yao-Shen Chen ${ }^{1}$ \\ 'Section of Infectious Diseases, Department of Medicine, Kaohsiung Veterans General Hospital, Kaohsiung, Taiwan and National Yang-Ming \\ University, Taipei, Taiwan; ${ }^{2}$ Department of Parasitology and Graduate Institute of Medicine, Kaohsiung Medical University, Kaohsiung, Taiwan
}

\begin{abstract}
Steroids are commonly used in patients with eosinophilic meningitis caused by $A$. cantonensis infections. The mechanism steroids act on eosinophilic meningitis remains unclear. In this mouse experiments, expressions of 14-3-3 isoform $\beta$ and $\gamma$ proteins significantly increased in the CSF 2-3 weeks after the infection, but not increasedin the dexamethasone-treated group. Expression of 14-3-3 $\beta, \gamma, \varepsilon$, and $\theta$ isoforms increased in brain meninges over the 3-week period after infection and decreased due to dexamethasone treatment. In conclusion, administration of dexamethasone in mice with eosinophilic meningitis decreased expressions of 14-3-3 isoform proteins in the CSF and in brain meninges.
\end{abstract}

Key words: Angiostrongylus cantonensis, dexamethasone, eosinophile, meningitis, 14-3-3 isoform

\section{INTRODUCTION}

Angiostrongylus cantonensis, also known as rat lungworm, was first described in Tainan, Taiwan in the 1940s. It is the main nematode causing eosinophilic meningitis or meningoencephalitis in Southeast Asia and the Pacific area [1-5]. Humans are infected with $A$. cantonensis by ingesting freshwater, terrestrial snails, slugs and other intermediate or paratenic hosts [58]. When this parasitic infection occurs in humans and mice (non-permissive hosts), the development of the larvae will stop at the young-adult worm stage in the brain and induce eosinophilia in blood and especially in cerebrospinal fluid (CSF) [8-13]. Previous studies have shown that transmigration of eosinophils to CSF [13], brain apoptosis [11] and the overexpression of CSF 14-3-3 $\beta$ [12] are associated with blood brain barrier (BBB) dysfunction in mice and humans infected with A. cantonensis.

In the brain, there are 7 isoforms $(\beta, \varepsilon, \gamma, \eta, \zeta, \tau / \theta$, and $\sigma)$ of 14-3-3 protein, and they modulate the action of proteins that

- Received 3 February 2019, revised 9 April 2019, accepted 9 April 2019

*Corresponding author (hctsai1011@yahoo.com.tw)

(c) 2019, Korean Society for Parasitology and Tropical Medicine

This is an Open Access article distributed under the terms of the Creative Commons Attribution Non-Commercial License (http://creativecommons.org/licenses/by-nc/4.0) which permits unrestricted non-commercial use, distribution, and reproduction in any

medium, provided the original work is properly cited. are involved in neurodegeneration, apoptosis, cell cycle control and signal transduction [14]. High levels of the 14-3-3 $\eta$ isoform have been reported in brain tissue, especially in Purkinje cells in the cerebellum [15]. High levels of 14-3-3 $\beta$ and $\gamma$ isoforms have also been reported, with the $\gamma$ isoform being more brain specific [16-18]. In normal murine brains, the $\beta, \gamma$, $\eta$, and $\zeta$ isoforms are distributed mainly in particular anatomical nuclei in the neuronal cell bodies. However, differences in the location of individual isoforms have been reported, with the $\tau$ isoform being found only in the hippocampus and medulla, and the $\varepsilon$ isoform being found throughout grey matter of the central nervous system (CNS) [19]. The presence of the 14-3-3 protein in CSF has been reported in individuals with Creutzfeldt-Jakob disease (CJD) [20], possibly due to disruption of neurons and leakage of brain parenchymal proteins into the CSF [21]. The 14-3-3 protein has also been found in CSF specimens obtained from patients with various neurological diseases, including bacterial and parasitic meningitis [22,23]. Previous research has demonstrated that the 14-3-3 protein is a neuropathologic disease marker that can be used to monitor neuronal damage in bacterial meningitis [23]. Although the lack of specificity limits the use of the 14-3-3 protein as a specific marker, its value as an indicator of BBB damage could be used to monitor the evolution of eosinophilic meningitis.

Steroids have been used as adjunctive treatment for eosino- 
philic meningitis. In the studies of Chotmongkol et al. [24] and Sawanyawisuth et al. [25], 2-week and 1-week courses of prednisolone were found to be beneficial in the treatment of headaches in patients with eosinophilic meningitis. In our previous study, we found that the beneficial effect of steroids on eosinophilic meningitis caused by A. cantonensis infection was mediated by the down-regulation of 14-3-3 $\beta$ protein expression in CSF and a reduction in BBB dysfunction [12]. In this study, we investigated the effect of dexamethasone treatment on dynamic changes of various 14-3-3 isoform protein expressions in parasitic eosinophilic meningitis, correlations with the severity of BBB damage and brain IHC findings, and reviewed the literature.

\section{MATERIAL AND METHODS}

\section{Ethics statement}

The Animal Committee of our hospital approved the study protocol. The animal studies were conducted in strict adherence with the recommendations from Taiwan's Animal Protection Act.

\section{Infection of Balb/c mice and intraperitoneal steroid injections}

The detailed experimental procedures have been described in a previous study [12]. Briefly, $40 \mathrm{Balb} / \mathrm{c}$ mice (5 groups, 8 in each group with an equal number of males and females), aged 6-7 weeks, were raised and maintained in an air-conditioned animal facility. Third-stage A. cantonensis larvae were harvested according to a previously described method [13]. The mice were orally infected with $50 \mathrm{~A}$. cantonensis L3 via an orogastric tube after slight ketamine anesthesia, and then 8 mice were sacrificed every week for 3 consecutive weeks after infection until the end of the study. Dexamethasone at a dose of $500 \mu \mathrm{g} /$ $\mathrm{kg} /$ day was injected intraperitoneally from the 7th day post infection (dpi) until the end of the study (21 dpi). The total duration of dexamethasone treatment was 2 weeks.

\section{Collection of serum and CSF sampes}

Blood and CSF samples were collected according to a previously published protocol [12].

\section{Measurement of permeability of the BBB using Evans blue}

A total of $200 \mu \mathrm{l}$ of $2 \%$ (w/v) Evans blue solution in PBS was injected into the tail vein of the Balb/c mice according to our previous study [22]. One hour later, the mice brains were removed after anesthesia with ketamine, and ground with 1.0 $\mathrm{ml}$ PBS. The extracts were then centrifuged, and the optical density of the supernatants was read at $595 \mathrm{~nm}$ using a colorimeter as described previously [22].

\section{Measurement of 14-3-3 protein concentrations in CSF/ serum/brain homogenates by Western blotting}

The detailed study procedure has been described in a previous study [22]. Briefly, whole brains were homogenized in lysis buffer and then centrifuged at $12,000 \mathrm{~g}$ for $15 \mathrm{~min}$ at $4^{\circ} \mathrm{C}$. The supernatants were then diluted and heated to $90^{\circ} \mathrm{C}$ for 10 $\mathrm{min}$. The supernatants were then separated by electrophoresis on 10\% Bis-Tris gels in SDS buffer. After transfer, the polyvinylidene fluoride (PVDF) membranes were probed at room temperature with mouse monoclonal IgG for pan 14-3-3 and rabbit polyclonal IgG antibody for 14-3-3 $\beta, \varepsilon, \gamma, \eta, \zeta, \tau, \theta$, and $\sigma$ (Santa Cruz Biotech, Santa Cruz, California, USA), followed by goat anti-rabbit horseradish peroxidase (HRP)-conjugated antibody (Santa Cruz Biotech). The blots were developed using an enhanced chemiluminescent system (Amersham; GE Healthcare Life Sciences). Densitometric values were obtained using a computer-assisted laser scanner (GS-710 Calibrated Imaging Densitometry; Bio-Rad, Hercules, California, USA).

\section{Immunohistochemical staining of 14-3-3 protein}

To identify the cellular localization of 14-3-3 in the brain meninges, coronal sections of brain tissue were immunohistochemically stained with a polyclonal antibody directed against the 14-3-3 proteins. The detailed study protocol was a modification of our previous study [12]. Paraffin-embedded brain tissue sections $(4 \mu \mathrm{m})$ of mouse brains were air dried, deparaffinized, rehydrated then heated to retrieve antigens. Endogenous peroxidase was quenched with $3 \% \mathrm{H}_{2} \mathrm{O}_{2}$ for $10 \mathrm{~min}$, and then sections were blocked with a blocking solution for 30 $\min$. The sections were then incubated either with mouse monoclonal IgG for pan 14-3-3 and rabbit polyclonal IgG antibody for 14-3-3 $\beta, \varepsilon, \gamma, \eta, \zeta, \tau, \theta$ or $\sigma$ with blocking solution for $60 \mathrm{~min}$ at room temperature. A secondary biotinylated multilink antibody was then added for $30 \mathrm{~min}$. After washing, streptavidin-horseradish peroxidase was applied for $20 \mathrm{~min}$, followed by diaminobenzidine tetrahydrochloride in buffer for 10 min (Novocastra ${ }^{\mathrm{TM}}$ Polymer Detection System; Leica Biosystems, Newcastle Upon Tyne, UK). 


\section{Statistical analysis}

The concentrations of Evans blue and 14-3-3 isoform proteins in the different groups were compared using the nonparametric Kruskal-Wallis test. The Mann Whitney U test was used to compare changes in 14-3-3 isoform proteins for every week relative to the control or steroid treatment groups. All of the experiments were done in triplicate. All results are presented as medium and range. A $P$-value less than 0.05 was considered to be statistically significant.

\section{RESULTS}

\section{Permeability of the BBB}

The concentration of Evans blue in the mice brains was significantly higher 3 weeks after infection compared to the uninfected mice $(P=0.022)$. The intensity of Evans blue in the brains of mice treated with dexamethasone for 2 weeks was significantly lower than that in the mice that had been infected for 3 weeks. Therefore, BBB dysfunction was more severe in the mice 3 weeks after infection compared to the uninfected mice (Fig. 1).

\section{4-3-3 isoforms in CSF, serum and brain homogenates of mice}

Concentrations of 14-3-3 isoforms in CSF/serum/brain homogenates is shown in Fig. 2. There were significant increases in 14-3-3 isoform proteins $\beta$ and $\gamma$ in the CSF in the 3rd week after infection. Dexamethasone treatment for 2 weeks (7th to 21 st dpi) significantly decreased the expressions of 14-3-3 isoform proteins $\beta$ and $\gamma$ in the CSF. However, the 14-3-3 isoform protein expressions in the brain homogenates and serum did not show any significant changes in the following weeks of infection or after dexamethasone treatment (Fig. 3).

\section{4-3-3 isoforms in the meninges of mice brains}

The IHC studies for 14-3-3 isoform proteins in brain meninges are shown in Fig. 4. There were significant increases in the concentrations of 14-3-3 isoform proteins $\beta, \gamma, \varepsilon$, and $\theta$ in the brain meninges in the 2nd and 3rd weeks post infection compared to the controls and 1st week post infection. Dexamethasone treatment significantly decreased the expressions of those 14-3-3 isoform proteins, and the decrease of 14-3-3

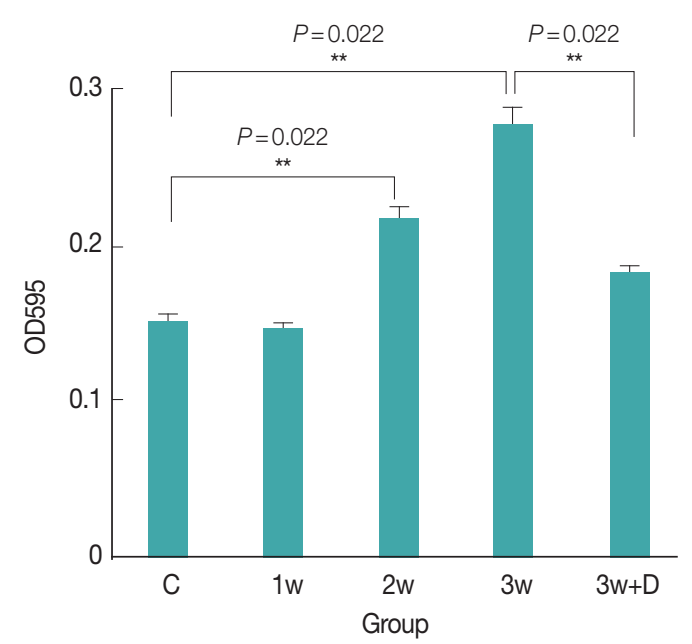

Fig. 1. Change of Evans blue concentrations in the brain homogenates of mice with $A$. cantonensis infection. C (Control), no parasitic infection; 1w, 1 week post infection; 2w, 2 weeks post infection; $3 w, 3$ weeks post infection; 3w+D (Dex), mice given dexamethasone for 2 weeks.
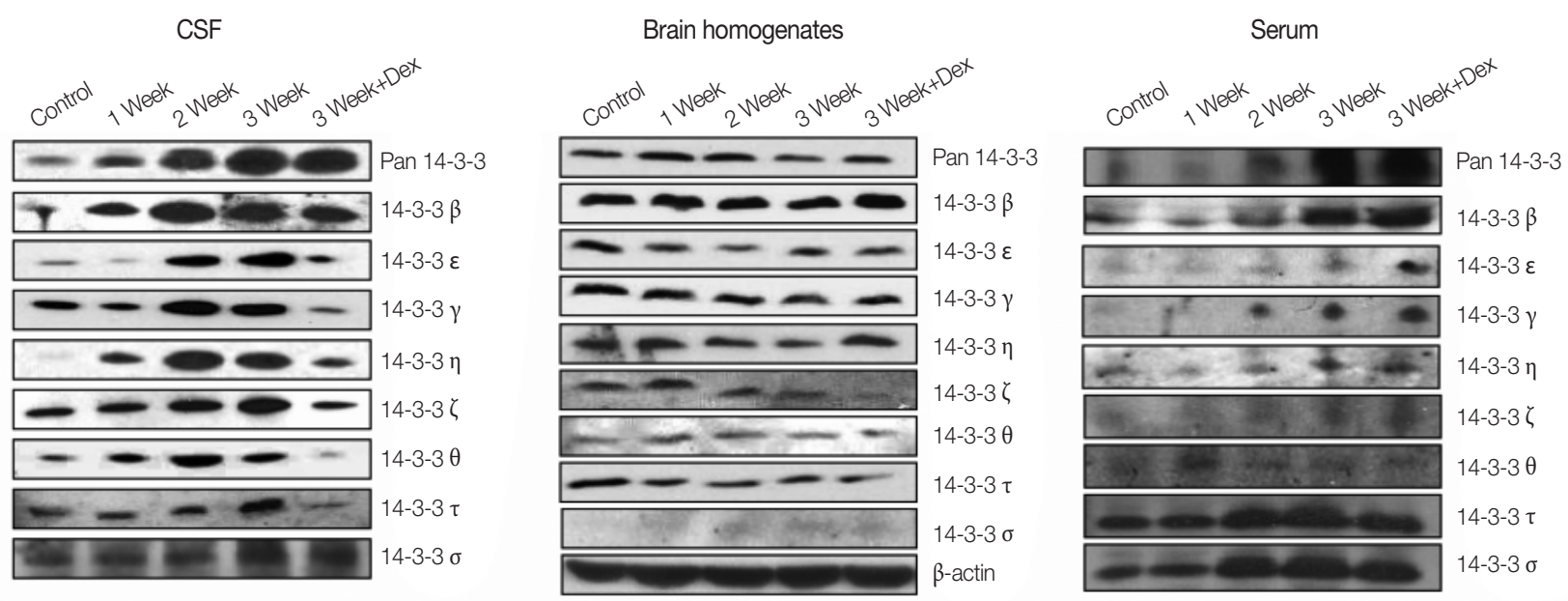

Fig. 2. Western blottings of 14-3-3 isoforms in CSF/brain homogenates/serum in mice with A. cantonensis infection. 

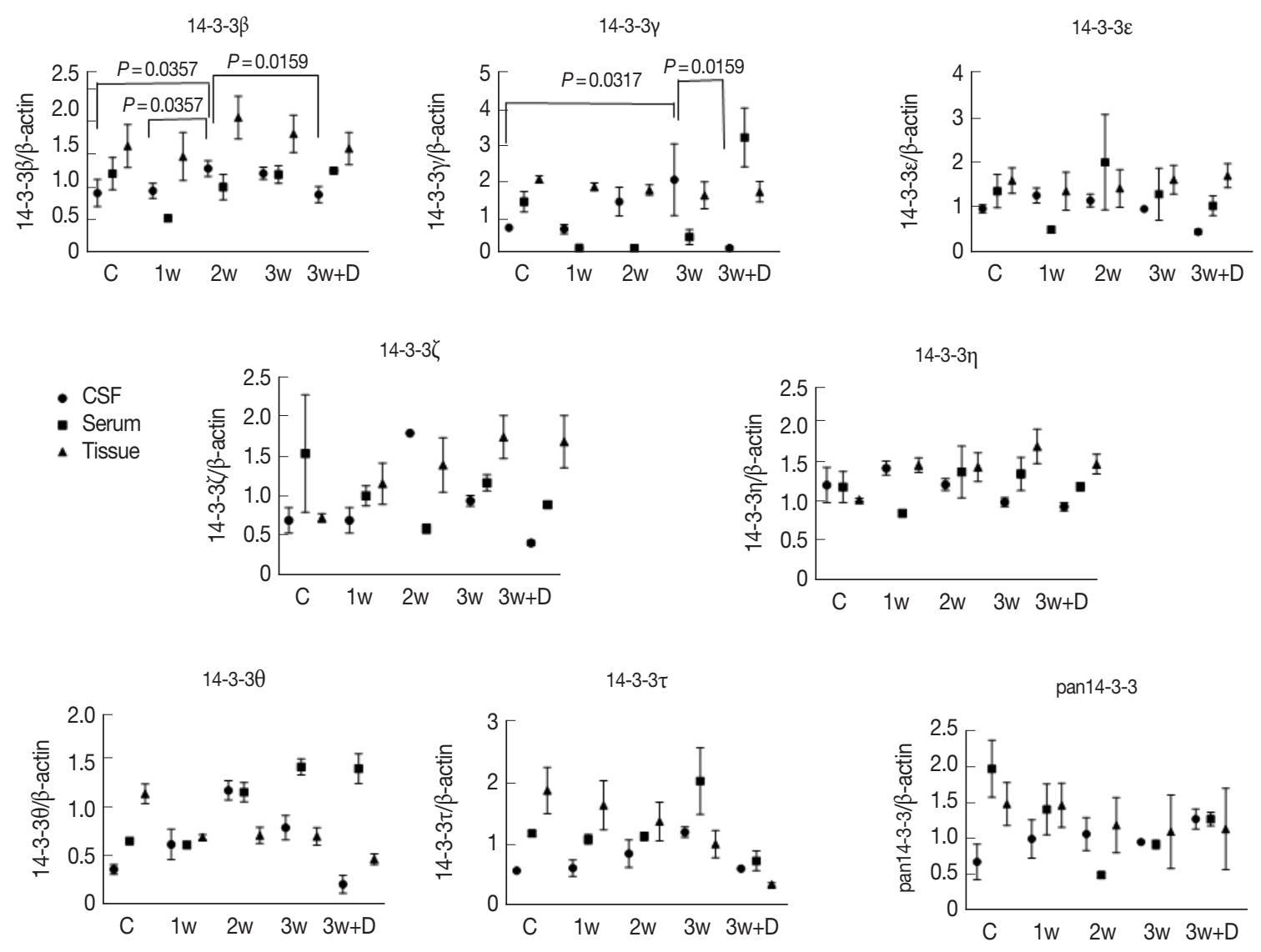

Fig. 3. Quantitative presentation of 14-3-3 isoforms in brain homogenate.

isoform proteins in brain meninges were isotype specific.

\section{DISCUSSION}

Our results showed a significant increase in 14-3-3 isoform proteins $\beta$ and $\gamma$ in CSF in the 2nd and 3rd weeks post infection compared to the controls and 1st week post infection, which was consistent with the severity of BBB damage as assessed by Evans blue assay. Dexamethasone treatment significantly decreased the amounts of 14-3-3 isoform proteins $\beta$ and $\gamma$ in CSF. Dexamethasone treatment significantly decreased the expressions 14-3-3 isoform proteins and Evans blue staining in the CSF and brain meninges. Steroid treatment has been shown to improve capillary function in the CSF of patients with multiple sclerosis by increasing levels of tissue inhibitors of matrix metalloproteinases (MMPs) and reducing activity of urokinase-type plasminogen activators and MMP-9 [26]. Steroid treatment in eosinophilic meningitis caused by A. cantonensis infection has been shown to partially inhibit the function of plasminogen activators and inflamma- tion [27]. In addition, steroid treatment of eosinophilic meningitis has been shown to improve intracranial pressure and also decrease neurological symptoms due to the inflammatory consequences of migrating and dying worms [28]. IL-5, a hemopoietic factor, has been shown to stimulate the proliferation, differentiation, and maturation of eosinophils [29], and an increased CSF IL-5 level and eosinophilia have been reported in eosinophilic meningitis caused by A. cantonensis infection $[13,30]$. Steroids have been found to induce the apoptosis of eosinophils [31] and inhibit brain apoptosis in mice with eosinophilic meningitis [11]. Taken together, the beneficial effects of steroids on eosinophilic meningitis caused by A. cantonensis infection in this study may have been mediated by improvements in BBB dysfunction, inhibition of the expressions of plasminogen activators and inflammation, down-regulation of 14-3-3 isoform proteins in the CSF, inhibition of mice brain apoptosis, and other unidentified mechanisms.

In the 1970s, John et al. [32] demonstrated how infective L3 larvae migrate from the gastrointestinal tract to the CNS via a hematogenous route in an animal model of eosinophilic 


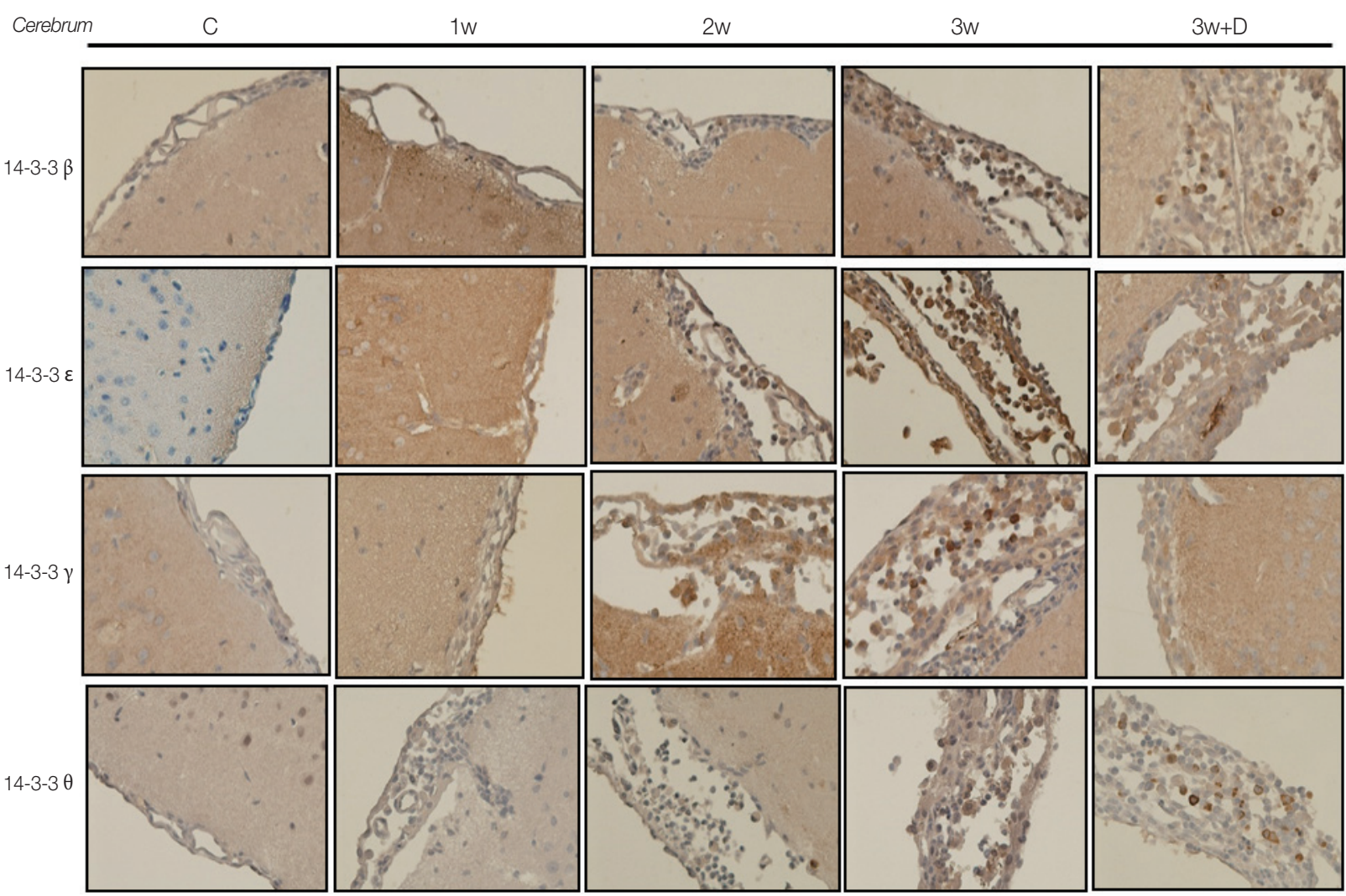

Fig. 4. Immunohistochemical stainings showed 14-3-3 $\beta, \gamma, \varepsilon$, and $\theta$ isoforms in the cerebrum.

meningitis caused by A. cantonensis infection. At approximately 10 days after parasitic infection, larvae mainly appeared in the subarachnoid spaces of posterior fossa structures and then gradually spread to the cerebral areas. They remained in the brain from 8 days to several weeks, and provoked a severe inflammatory reaction and direct or mechanical injury [32]. This time course of brain destruction is consistent with the present study, in that the most severe BBB damage occurred in the 2nd and 3rd weeks post infection with simultaneous increases in the expressions of 14-3-3 isoform proteins $\beta$ and $\gamma$ in the CSF and meninges.

The 14-3-3 proteins are highly conserved proteins that play important roles in regulating many cell functions including the onset of cell differentiation, maintenance of the cell cycle, preventing apoptosis, and DNA repair [33]. The highest tissue concentrations of 14-3-3 proteins occur in the brain, and constitute approximately $1 \%$ of total soluble protein [34]. In addition to their possible roles in neuronal function, 14-3-3 proteins are also involved in the pathophysiology of various neurological disorders [12,20,23,35,36]. Different 14-3-3 isoforms have been implicated in the regulation of many intracellular signaling pathways [33]. Furthermore, the 14-3-3 proteins have been found to regulate the mitogen-activated protein kinase (MAPK) pathway [37]. There are 7 14-3-3 isoforms: $\beta, \varepsilon$, $\gamma, \eta, \zeta, \tau / \theta$, and $\sigma$. The 14-3-3 proteins regulate apoptotic signals. Specifically, the 14-3-3 $\theta$ isoform is bound to $\mathrm{Bcl}-2$ associated X protein (Bax) in the cytoplasm, which undergoes dissociation from 14-3-3 $\theta$ during apoptosis to induce apoptotic changes of the mitochondria [37].

A previous study demonstrated that the $14-3-3 \beta$ protein was a neuropathologic marker that could be used to monitor neuronal damage in patients with bacterial and eosinophilic meningitis $[12,23]$. Increased pan 14-3-3 and 5 isoform proteins $(\beta, \gamma, \varepsilon, \eta, \zeta)$ have also been reported in the CSF of adults with bacterial meningitis [17]. Elevated CSF 14-3-3 $\gamma$ protein levels have been reported in HIV-negative patients with cryptococcus meningitis, with no changes in the levels with shortterm treatment [18]. In addition, Morassutti et al. [38] reported that the 14-3-3 proteins reacted with 31-kDa antigens in $A$. cantonensis infection.

In the present study, we demonstrated that the dynamic changes in 14-3-3 isoform proteins $\beta$ and $\gamma$ in the mice CSF 
and brain meninges were correlated with the dynamic changes of BBB dysfunction, as indicated by the Evans blue assay. Not all of the 14-3-3 isoform proteins in the CSF/brain meninges similarly increased 2-3 weeks post infection nor similarly decreased after dexamethasone treatment. This is in agreement with the previous findings that $14-3-3 \gamma$ is brain specific and $14-3-3 \beta$ is a neuropathologic marker. Further studies are needed to clarify this phenomenon.

Our results suggest that 14-3-3 proteins could be useful markers of neuronal damage, and that the presence of 14-3-3 isoform proteins in CSF may be the consequence of disruption of the BBB caused by parasitic eosinophilic meningitis, since it has been shown that parasitic infections can induce up-regulation of MMP-9 [39], vascular endothelial growth factor [40] and hepatocyte growth factor [41], and that the infection contributes to damage of meningeal blood vessel membranes. This change in the cerebral vasculature could account for the leakage of 14-3-3 proteins, which are primarily CNS proteins, into the CSF circulation.

Further studies are needed to elucidate why some of the 143-3 isoform proteins in the mice brains did not significantly increase after infection or decrease after dexamethasone treatment. Possible explanations include the uneven distribution of 14-3-3 isoform proteins and the unpredictable mechanical injury caused by the parasitic infection in the mice brains, as well as the semi-quantitative nature of the Western blot analysis. The only small increase in serum 14-3-3 isoform proteins after infection and the poor effect of dexamethasone treatment in decreasing the serum expressions of 14-3-3 isoform proteins might be related to lower serum steroid concentrations caused by intraperitoneal injections and the semi-quantitative nature of the Western blot analysis.

There are several limitations to this study. First, the Evans blue method has limited sensitivity for monitoring BBB dysfunction. The sensitivity would be improved if the albumin levels in serum and CSF were simultaneously quantified and the CSF/serum albumin ratio calculated. Second, the animal samples were analyzed using the semi-quantitative Western blot method, which may have decreased the sensitivity and specificity. Finally, the data obtained in the mice model may not be applied to human infection.

In conclusion, the current study showed that the mice with eosinophilic meningitis with increasing 14-3-3 isoform proteins $\beta$ and $\gamma$ in CSF and brain meninges tended to have more severe BBB dysfunction as evidenced by Evans blue assay. In addition, there were remarkable reductions in 14-3-3 protein isoforms $\beta$ and $\gamma$ in the CSF and $\beta, \gamma, \varepsilon$, and $\theta$ isoforms in the brain meninges after 2 weeks of dexamethasone treatment. These results highlight the role of 14-3-3 proteins in eosinophilic meningitis caused by $A$. cantonensis.

\section{ACKNOWLEDGMENTS}

We would like to thank Pei-Yun Chou and I-Tzu Chen for their assistance in the animal study. The animal studies were conducted in strict accordance with the recommendations from Taiwan's Animal Protection Act. The Animal Committee of Kaohsiung Veterans General Hospital approved the study protocol.

The datasets used and/or analyzed during the current study are available from the corresponding author on reasonable request.

This work was supported by grants from Kaohsiung Veterans General Hospital (VGHKS 100-046) and the Ministry of Science and Technology, Republic of China (MOST 104-2320-B075B-004). The funders had no role in study design, data collection and analysis, decision to publish, or preparation of the manuscript.

\section{CONFLICT OF INTEREST}

The authors declare no conflict of interest related to this study.

\section{REFERENCES}

1. Beaver PC, Rosen L. Memorandum on the first report of Angiostrongylus in man, by Nomura and Lin, 1945. Am J Trop Med Hyg 1964; 13: 589-590.

2. Punyagupta S, Juttijudata P, Bunnag T. Eosinophilic meningitis in Thailand. Clinical studies of 484 typical cases probably caused by Angiostrongylus cantonensis. Am J Trop Med Hyg 1975; 24: 921-931.

3. Rosen L, Chappell R, Laqueur GL, Wallace GD, Weinstein PP. Eosinophilic meningoencephalitis caused by a metastrongylid lung worm of rats. JAMA 1962; 179: 620-624.

4. Yii CY. Clinical observations on eosinophilic meningitis and meningoencephalitis caused by Angiostrongylus cantonensis on Taiwan. Am J Trop Med Hyg 1976; 25: 233-249.

5. Nishimura K, Mogi M, Okazawa T, Sato Y, Toma H, Wakibe H. Angiostrongylus cantonensis infection in Ampullarium canaliculatus in Kyusu, Japan. Southeast Asian J Trop Med Pub Health 1986; 
17: 595-600.

6. Richards CS, Merritt JW. Studies on Angiostrongylus cantonensis in molluscan intermediate hosts. J Parasitol 1967; 53: 382-388.

7. Yen CM, Chen ER, Cheng CW. A survey of Ampullarium canaliculatus for natural infection of Angiostrongylus cantonensis in south Taiwan. J Trop Med Hyg 1990; 93: 347-350.

8. Tsai HC, Liu YC, Kunin CM, Lee SS, Chen YS, Lin HH, Tsai TH, Lin WR, Huang CK, Yen MY, Yen CM. Eosinophilic meningitis caused by Angiostrongylus cantonensis: report of 17 cases. Am J Med 2001; 111: 109-114.

9. Tsai HC, Liu YC, Kunin CM, Lai PH, Lee SS, Chen YS, Wann SR, Lin WR, Huang CK, Ger LP, Lin HH, Yen MY. Eosinophilic meningitis caused by Angiostrongylus Cantonensis associated with eating raw snails: correlation of brain magnetic resonance imaging scans with clinical findings. Am J Trop Med Hyg 2003; 68: 281285.

10. Tsai HC, Lee SS, Huang CK, Yen CM, Chen ER, Liu YC. Outbreak of eosinophilic meningitis associated with drinking raw vegetable juice in southern Taiwan. Am J Trop Med Hyg 2004; 71: 222-226.

11. Tsai HC, Lee BY, Yen CM, Wann SR, Lee SS, Chen YS. Dexamethasone inhibits brain apoptosis in mice with eosinophilic meningitis caused by Angiostrongylus cantonensis infection. Parasit Vectors 2015; 8: 200.

12. Tsai HC, Lee BY, Yen CM, Wann SR, Lee SS, Chen YS, Tai MH. Dexamethasone downregulated the expression of CSF 14-3-3 $\beta$ protein in mice with eosinophilic meningitis caused by Angiostrongylus cantonensis infection. Acta Trop 2014; 131: 98-103.

13. Lee JD, Tsai LY, Chen CH, Wang JJ, Hsiao JK, Yen CM. Bloodbrain barrier dysfunction occurring in mice infected with Angiostrongylus cantonensis. Acta Trop 2006; 97: 204-211.

14. Berg D, Holzmann C, Riess O. 14-3-3 proteins in the nervous system. Nat Rev Neurosci 2003; 4: 752-762.

15. Watanabe $M$, Isobe $T$, Okuyama T, Ichimura T, Kuwano R, Takahashi Y, Kondo H. Molecular cloning of cDNA to rat 14-3-3 eta chain polypeptide and the neuronal expression of the mRNA in the central nervous system. Brain Res Mol Brain Res 1991; 10: 151-158.

16. Sobe T, Ichimura T, Sunaya T, Okuyama T, Takahashi N, Kuwano $\mathrm{R}$, Takahashi Y. Distinct forms of the protein kinase-dependent activator of tyrosine and tryptophan hydroxylases. J Mol Biol 1991; 217: 125-132.

17. Lu CH, Chang WN, Chang HW, Chung KJ, Tsai HC, Wang HC, Chen SS, Chuang YC, Huang CR, Tsai NW, Chiang YF. The value of serial cerebrospinal fluid 14-3-3 protein levels in adult community-acquired bacterial meningitis. QJM 2008; 101: 225-230.

18. Chang WN, Lu CH, Huang CR, Chuang YC, Tsai NW, Chen SF, Chang CC, Wang HC. Cerebrospinal fluid 14-3-3-gamma protein level in eight HIV-negative cryptococcal meningitis adults. Eur J Neurol 2008; 15: 428-430.

19. Baxter HC, Liu WG, Forster JL, Aitken A, Fraser JR. Immunolocalisation of 14-3-3 isoforms in normal and scrapie-infected murine brain. Neuroscience 2002;109: 5-14.
20. Hsich G, Kenney K, Gibbs CJ, Lee KH, Harrington MG. The 143-3 Brain protein in cerebrospinal fluid as a marker for transmissible spongiform encephalopathies. N Engl J Med 1996; 335: 924-930.

21. Aksamit AJ. Cerebrospinal fluid 14-3-3 protein: variability of sporadic Creutzfeldt-Jakob disease, laboratory standards, and quantitation. Arch Neurol 2003; 60: 803-804.

22. Tsai HC, Huang YL, Chen YS, Yen CM, Tsai R, Lee SS, Tai MH. 14-3-3 3 protein expression in eosinophilic meningitis caused by Angiostrongylus cantonensis infection. BMC Res Notes 2014; 7: 97.

23. Bonora S, Zanusso G, Raiteri R, Monaco S, Rossati A, Ferrari S, Boffito M, Audagnotto S, Sinicco A, Rizzuto N, Concia E, Di Perri G. Clearance of 14-3-3 protein from cerebrospinal fluid heralds the resolution of bacterial meningitis. Clin Infect Dis 2003; 36: 1492-1495.

24. Chotmongkol V, Sawanyawisuth K, Thavornpitak Y. Corticosteroid Treatment of Eosinophilic Meningitis. Clin Infect Dis 2000; 31: 660-662.

25. Sawanyawisuth K, Limpawattana P, Busaracome P, Ninpaitoon B, Chotmongkol V, Intapan PM, Tanawirattananit S. A 1-week course of corticosteroids in the treatment of eosinophilic meningitis. Am J Med 2004; 117: 802-803.

26. Rosenberg GA, Dencoff JE, Correa N Jr, Reiners M, Ford CC. Effect of steroids on CSF matrix metalloproteinases in multiple sclerosis: relation to blood-brain barrier injury. Neurology 1996; 46: 1626-1632.

27. Tu WC, Lai SC. Angiostrongylus cantonensis: efficacy of albendazole-dexamethasone co-therapy against infection-induced plasminogen activators and eosinophilic meningitis. Exp Parasitol 2006; 113: 8-15.

28. Pien FD, Pien BC. Angiostrongylus cantonensis eosinophilic meningitis. Int J Infect Dis 1999; 3: 161-163.

29. Lopez AF, Sanderson CJ, Gamble JR, Campbell HD, Young IG, Vadas MA. Recombinant human interleukin 5 is a selective activator of human eosinophil function. J Exp Med 1988; 167: 219224.

30. Intapan PM, Kittimongkolma S, Niwattayakul K, Sawanyawisuth $\mathrm{K}$, Maleewong W. Cerebrospinal fluid cytokine responses in human eosinophilic meningitis associated with angiostrongyliasis. J Neurol Sci 2008; 267: 17-21.

31. Rothenberg ME. Eosinophilia. N Engl J Med 1998; 338: 15921600.

32. John DT, Martinez AJ. Animal model of human disease. Central nervous system infection with the nematode Angiostrongylus cantonenis. Animal model:eosinophilic meningoencephalitis in mice infected with Angiostrongylus cantonensis. Am J Pathol 1975; 80: 345-348.

33. Berg D, Holzmann C, Riess O. 14-3-3 proteins in the nervous system. Nat Rev Neurosci 2003; 4: 752-762.

34. Boston PF, Jackson P, Thompson RJ. Human 14-3-3 Protein: Radioimmunoassay, Tissue Distribution, and Cerebrospinal Fluid Levels in Patients with Neurological Disorders. J Neurochem. 1982; 38: 1475-1482. 
35. Collins S, Boyd A, Fletcher A, Gonzales M, McLean CA, Byron K, Masters CL. Creutzfeldt-Jakob disease: diagnostic utility of 14-33 protein immunodetection in cerebrospinal fluid. J Clin Neurosci 2000; 7: 203-208.

36. Irani DN, Kerr DA. 14-3-3 protein in the cerebrospinal fluid of patients with acute transverse myelitis. Lancet 2000; 355: 901.

37. Fu H, Subramanian RR, Masters SC. 14-3-3 Proteins: structure, function, and regulation. Annu Rev Pharmacol Toxicol 2000; 40: 617-647.

38. Morassutti AL, Levert K, Perelygin A, da Silva AJ, Wilkins P, Graeff-Teixeira C. The 31-kDa antigen of Angiostrongylus cantonensis comprises distinct antigenic glycoproteins. Vector Borne Zoonotic Dis 2012; 12: 961-968.

39. Tsai HC, Chung LY, Chen ER, Liu YC, Lee SS, Chen YS, Sy CL,
Wann SR, Yen CM. Association of matrix metalloproteinase-9 and tissue inhibitors of metalloproteinase- 4 in cerebrospinal fluid with blood-brain barrier dysfunction in patients with eosinophilic meningitis caused by Angiostrongylus cantonensis. Am J Trop Med Hyg 2008; 78: 20-27.

40. Tsai HC, Liu YC, Lee SS, Chen ER, Yen CM. Vascular endothelial growth factor is associated with blood brain barrier dysfunction in eosinophilic meningitis caused by Angiostrongylus cantonensis infection. Am J Trop Med Hyg 2007; 76: 592-595.

41. Tsai HC, Huang YL, Liu YC, Wann SR, Lee SS, Chen ER, Yen CM, Tai MH, Shi MH, Chen YS. Dynamic changes of hepatocyte growth factor in eosinophilic meningitis caused by Angiostrongylus cantonensis infection. Am J Trop Med Hyg 2009; 80: 980-982. 